\title{
The use of high tumescent power assisted liposuction in the treatment of Madelung's collar
}

\author{
Henryk Witmanowski ${ }^{1,2}$, Łukasz Banasiak ${ }^{1}$, Grzegorz Kierzynka ${ }^{1}$, Jarosław Markowicz ${ }^{1}$, Jerzy Kolasiński ${ }^{1}$, \\ Katarzyna Błochowiak ${ }^{3}$, Paweł Szychta ${ }^{1,4}$
}

${ }^{1}$ Department of Plastic, Reconstructive and Aesthetic Surgery, Medical College in Bydgoszcz, Nicolaus Copernicus University in Torun, Poland ${ }^{2}$ Department of Physiology, Poznan University of Medical Sciences, Poznan, Poland

${ }^{3}$ Department of the Oral Surgery, Poznan University of Medical Sciences, Poznan, Poland

${ }^{4}$ Department of Oncological Surgery and Breast Diseases, Polish Mother's Memorial Hospital-Research Institute, Lodz, Poland

Adv Dermatol Allergol 2017; XXXIV (4): 366-371

DOI: https://doi.org/10.5114/ada.2017.69319

Mild symmetrical lipomatosis (plural symmetrical lipomatosis, multiple symmetric lipomatosis - MSL), also known as Madelung's disease or Launois-Bensaude syndrome is a rare disease of unknown etiology, first described by Brodie in 1846, Madelung in 1888, and Launois with Bensaude in 1898 [1-3].

Madelung's disease incidence is $1: 250000$ [4]. Multiple symmetric lipomatosis occurs mainly in the inhabitants of the Mediterranean area and Eastern Europe, in males (male to female ratio is $20: 1$ ), aged 30-70 years, usually in conjunction with a heavy abuse of alcohol (up to $90 \%$ of cases) [4].

It is often associated with other disease entities liver disease, impaired glucose tolerance, hyperuricemia, hypothyroidism, hypertension, and hyperlipidemia [5] Most of the reported cases of this disease occur sporadically, although family forms with autosomal dominant or maternal mitochondrial inheritance formula are known [6-9].

The characteristic image of this disease is symmetrical subcutaneous adipose encapsulated tissue accumulation, which is slowly and continuously increasing in the neck, the upper part of the upper limb, pelvic rim, back and thighs. Two main types of the disease are described and classified on the basis of fat distribution: type I where lipomas gather around the neck, supraclavicular area and around the shoulder - giving the image of Madelung's collar or a pseudo-athletic figure, in type II fatty tissue develops under the skin evenly giving a picture of simple obesity with excessive fat deposits in the vicinity of the back, shoulders, hips, and upper thighs. Another classification distinguishes three types of body profiles in patients with Madelung's disease; type of cervical location, pseudo-athletic and gynecoid (abdomino-iliac) type [10].
The disease most commonly takes a proximal form which takes the following areas of the body with the following frequency: the genial area $92.3 \%$, cervical region $67.7 \%$, shoulder region $54.8 \%$, abdominal $45.2 \%$, chest $41.9 \%$, thigh and pelvic rim $32.3 \%[11,12]$. The peripheral type mainly locates on both sides at the level of the hands, feet and knees, this is definitely a rarer form of the disease. The mixed form is described very rarely. The specified central type is also engaged primarily around the lower torso, and intermediate parts of the legs. Typically for the Madelung's disease, the gluteal region and shanks are not occupied [13].

Moreover, an unsightly appearance of the changes, in some cases lipomas oppress and even infiltrate the space of adjacent structures, leading to compression syndromes of the upper respiratory tract - even life-threatening ones, the upper gastrointestinal tract, decreased mobility of the cervical spine and in the case of mediastinal location, the syndrome of superior vena cava.

The etiology and pathogenesis are not fully understood, among the most plausible theories one should mention a catecholamine-dependent lipid metabolism defect, mutations in mitochondrial DNA, and the primary peripheral neuropathy. In addition, there is evidence indicating that precursors of lipomas may be cells of brown adipose tissue.

One alternative hypothesis of the pathogenesis of MSL is a defect of brown adipose tissue (BAT), the residue of embryonic origin. The study of biology of fat cells in the MSL reveals a catecholamine-dependent defect in lipolysis, leading to the accumulation of triglycerides. Multiple symmetric lipomatosis adipocytes are capable of synthesis of thermogenin uncouple protein 1 (UCP-1), which is a selective marker of BAT (located in the mito-

Address for correspondence: Prof. Henryk Witmanowski MD, PhD, Department of Plastic, Reconstructive and Aesthetic Surgery, Medical College, Nicolaus Copernicus University, 9-11 M. Skłodowska-Curie St, 85-094 Bydgoszcz, Poland, phone: +48 52585 4017, e-mail: hewit7@wp.pl

Received: 21.06.2016, accepted: 26.07.2016. 
chondria of BAT). The function of UCP-1 is to generate heat without a tremor in the newborn. The MSL fat cells are not capable of epinephrine-dependent stimulation of UCP-1, so they are unable to consume the accumulated fat. Moreover, the lack of stimulation by epinephrine does not cause increased synthesis of nitric oxide, which has antiproliferative properties, thereby giving rise to an increase in adipose tissue $[6,14]$.

Madelung's disease association with mitochondrial dysfunction was also shown. The study revealed the dysfunction of mitochondria in diseased tissue fat, irregularities in the functioning of red muscle fibers and increased activity of cytochrome $\mathrm{C}$ in the respiratory muscles and decreased activity of three enzymes of the mitochondrial respiratory pathway (complex I- NADH, coenzyme Q10 reductase, complex III - coenzyme Q10, cytochrome C reductase, complex IV - cytochrome C oxidase). This may have an impact on mitochondrial lipid metabolism [15].

There is a connection with excessive alcohol consumption, depending on the source, $90 \%$ of cases is associated with alcohol abuse. The authors suggest that chronic alcohol consumption increases lipogenesis and reduces lipolysis. In addition, alcohol has a direct effect on mitochondrial metabolism and causes premature aging of mtDNA [16]. A mechanism for reducing the number and activity of $\beta$-adrenergic receptors seems significant, thus reducing the lipolytic processes and increasing lipogenesis $[14,17]$. Demyelinating alcohol-associated disorders of the peripheral nervous system may have an effect on $\beta$-adrenergic stimulation [14].

However, a significant part of cases of non-Mediterranean and non-Eastern Europe origin are women and children, and men without a history of alcohol abuse, which may indicate that alcohol is a factor promoting, rather than conditioning the occurrence of MSL [18].

Some researchers postulated that Madelung's disease is associated with polyneuropathy and not with alcoholism. Neuropathy has a range of sensory, motor and autonomic, the clinical manifestation of which may be neuropathic ulcers. The related study was performed as an analysis of sural nerve biopsies from the point of the existence of chronic peripheral neuronal loss, and showed no typical lesions for the alcoholic etiology - axonal degeneration and demyelination [19]. Damage to peripheral vegetative nerves widely affect the $\beta$-adrenergic stimulation.

From the histopathological point of view, MSL fat cells are smaller than normally present in adipose tissue, suggesting hyperplasia [14]. An increase of so-called tumor is also noted as the proliferation index, also described as a morphological intermediate form between lipoma and liposarcoma [7]. Isolated adipocytes have increased lipoprotein lipase activity, while pointing out to the defect of lipolysis stimulated by adrenergic receptors. The pathway of cAMP-dependent stimulation is maintained, which indicates a defect at the level of cellular receptors [19].
A 42-year-old patient came to the Department due to cervical disease manifestations in the form of so-called Madelung's collar. At the time of admission, the patient was $180 \mathrm{~cm}$ high and weighed $82 \mathrm{~kg}$. The patient was treated successfully by surgery earlier in the Department of Plastic, Reconstructive and Aesthetic Surgery of Medical College in Bydgoszcz in July 2013, because of scrotal lesion location, then about $2 \mathrm{~kg}$ of fat was removed. The results of laboratory tests found elevated levels of total cholesterol (281 mg/dl) and triglycerides (755 mg/dl). In an interview, hereditary involvement and addictions are not reported. At the moment, symmetrical changes are present in the abdomen, chin area, cheek and neck. In laboratory tests at the time of admission to the Department, a slight deviation from the norm in total cholesterol $(233 \mathrm{mg} / \mathrm{dl})$, high-density lipoprotein cholesterol (49 mg/dl), low-density lipoprotein cholesterol (151 mg/ dl) and triglycerides (164 mg/dl) was observed. The surgery was performed under general endotracheal anesthesia when lying on the back with the head deviation towards the rear. Before surgery, approximately one liter of tumescent liquid was prepared $-20 \mathrm{ml}$ of $2 \%$ lignocaine, 1 amp was added to $1000 \mathrm{ml}$ of $0.9 \% \mathrm{NaCl}$. Adrenaline at a dose of $0.5 \mathrm{mg}$ and $50 \mathrm{ml}$ of $8.4 \% \mathrm{NaHCO}_{3}$. By a microcut in the midline of the chin, using the multipoint cannula, between the muscle and the skin of the neck, the patient given approximately $400 \mathrm{ml}$ of fluid with medium pressure of about $200 \mathrm{~mm} \mathrm{Hg}$. Power assisted liposuction (PAL) performed with the cutting in the middle of the chin and with the symmetrical cuts in the area of the mandibular angle using a MicroAire device. A vibrating cannula $(3 \mathrm{~mm})$ of the "Mercedes type" was used and a total of about $400 \mathrm{ml}$ of adipose tissue was sucked. Fatty tissue removed was characterized with high density. Liposuction continued to reduce the "pinch test" from the initial results of approximately $4 \mathrm{~cm}$ to $1.5 \mathrm{~cm}$. After the treatment the patient was applied a pressure dressing and soft collar. The postoperative course was uneventful. The functional and aesthetic effect was satisfactory to both, the patient and the team. In the next stage enucleation of fatty tissues in the cervical and the cheek region using PAL is planned (Figures 1 and 2).

The disease can be easily diagnosed because of the characteristic changes in the distribution of fat: symmetrically, painless, encapsulated with no regression despite the use of caloric restrictions [20]. The massive symmetric fat deposition causes severe aesthetic distortion around the parotid region - "hamster cheeks", cervical region "horse collar" and neck - "buffalo hump" [21].

Madelung's disease diagnosis is primarily determined on the basis of a thorough clinical examination and analysis of the patient's history. The diagnosis is usually based on the characteristic clinical picture, ultrasound, often additionally supported by computed tomography (CT) or magnetic resonance (MRI). The CT and MRI are useful in assessing the distribution of excess fat, ultrasound does 

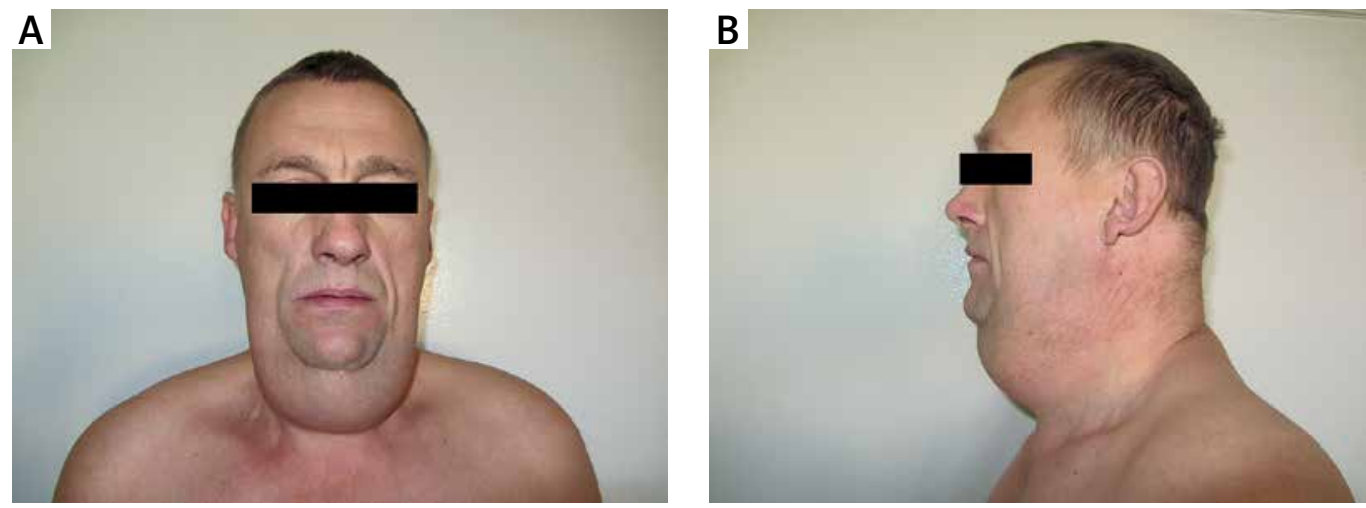

Figure 1. Patient 42 years old before power assisted liposuction
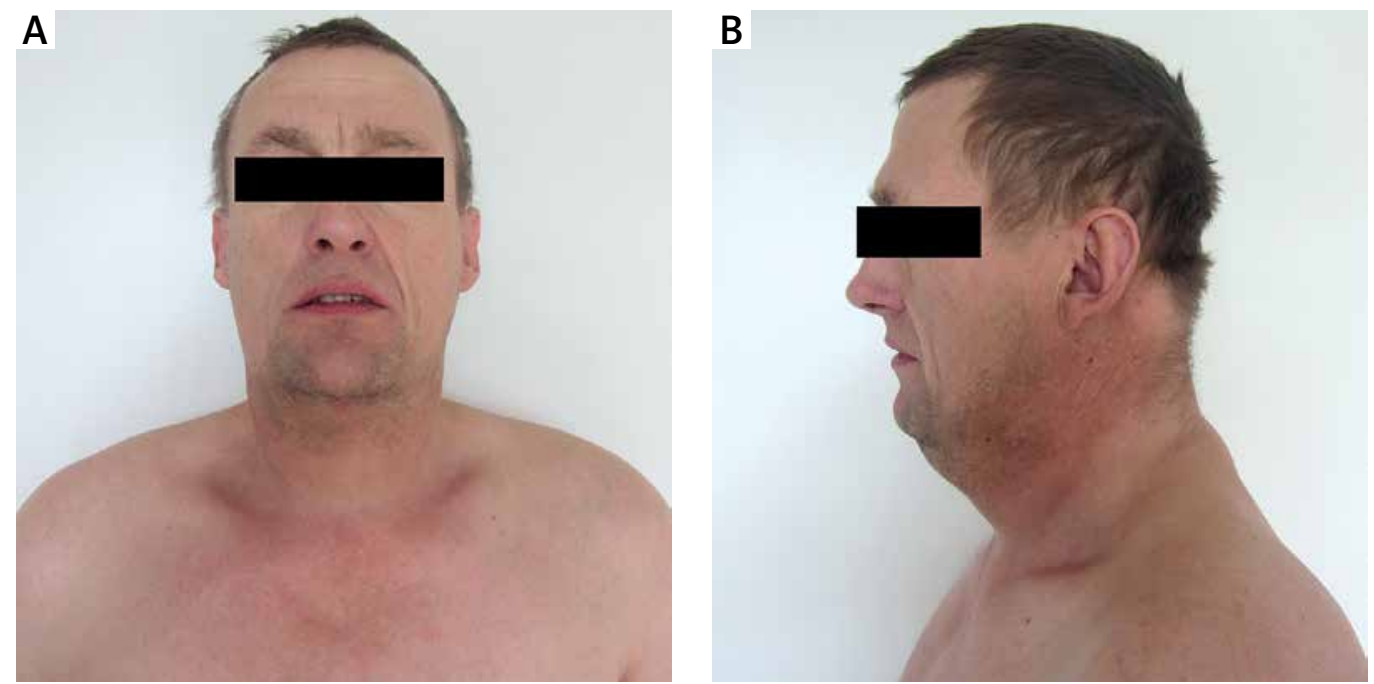

Figure 2. Patient 42 years old after power assisted liposuction

not give all the information. Diagnosis should be complemented by a routine chest $\mathrm{X}$-ray that can show abnormal symmetrical fatty mass in the mediastinum. The best diagnostic tool in the staging of local lesions and the degree of infiltration of adjacent tissues is MRI. This examination gives a full insight into the changes in local tissues and e.g. compression of the trachea, displacement of testis, in relation to fat mass and location of the vessels. It also offers the possibility to exclude any concomitant diseases, inter alia tumors [22]. Madelung's disease is often confused with other diseases; symmetrical arrangement of lipomas may lead to an erroneous diagnosis of simple obesity. For confirmation of the diagnosis, microscopic evaluation of the masses is necessary. Histologically, the cells obtained from the areas of changes are almost indistinguishable from normal adipose tissue, are only slightly smaller and multivacuolar [23].

One possible cause of incorrect initial diagnosis of this disease is probably the fact that there are no strict inclusion criteria as to the location of changes. The most significant change for the diagnosis is the simultaneous occurrence of lack of capsules along with the location recognized as typical [24]. In view of the extended time to disease progression, delay in diagnosis and incorrect diagnoses are more likely to be in the first phase of the disease, when symptoms are not fully visible, until the severity of the disease gives a characteristic grotesque appearance.

When determining a definitive diagnosis, in the differential diagnosis it is important to consider other more common disease entities such as simple obesity, angiolipoma, neurofibromatosis, capsulated lipomas, lipoblastomatosis, hibernoma, congenital infiltrating lipomatosis of the face, encephalocraniocutaneus lipomatosis, adiposarcoma and diseases of the salivary glands [25]. Lipomatosis of a similar nature is observed in HIVpositive people, it is associated with taking antiretroviral drugs known as protease inhibitors [23]. The differential diagnosis should take into account other disease enti- 
ties such as Cushing's syndrome, lymphomas, Dercum's disease or thyroid disorders [26, 27].

Characteristics of diseases to which attention should be paid in the differential diagnosis:

- Obesity - body mass index (BMI) over 30 or waist-hip ratio $(W H R)>0.9$ in men, $>0.7$ in women, the adipose tissue is distributed in a manner typical of sex, for males in the abdominal region, in females around the iliofemoral region [28].

- Angiolipoma - capsulated, benign and well-confined tumors with an excessive degree of vascular proliferation. They present in the form of painful nodules, usually around the elbows and back. The pain usually does not respond to painkillers. Histopathology reveals changes, encysted benign tumors, consisting of the small-caliber vessels with microemboli and the presence of varying amounts of mature adipose tissue [29].

- Neurofibromatosis - subcutaneous nodules composed of nerves and fibroblasts, patches of the skin in the coffee with milk color (café-au-lait) and pigmentary changes of the iris - Lisch nodules. Neurofibromas may be present in other locations. Changes tend to become malignant, moreover, this syndrome predisposes to cancers of the central nervous system (CNS) and adrenal glands [30].

- Lipoblastomatosis - fetal lipoma, a capsulated fatty tumor derived from fetal white adipose tissue, the change occurs almost exclusively in infants and young children, $90 \%$ of cases occur up to 3 years of age. Most of the changes occur on the limbs and trunk, the tumor is often confused with liposarcoma [31].

- Hibernoma - benign tumor derived from brown adipose tissue, usually located on the back, taking the form of a slightly haughty, brown change. Microscopically, the cells have multivacuolar cytoplasm and are wound with a network of blood vessels [32]

- Congenital infiltrating lipomatosis of face (CILoF) a rare congenital disease characterized by infiltration of soft tissue of the face, lipomas are encapsulated, occurs mostly in children, manifested by asymmetric changes including face [33].

- Familial multiple lipomatosis (HML, hereditary multiple lipomatosis, FML) - a hereditary syndrome of autosomal dominant inheritance with an incomplete penetrance. More common in males. It reveals the presence of many discrete capsulated lipomas. It occurs mainly on the trunk and extremities, extremely rarely on the head and shoulders. The number of tumors in the typical patient may vary considerably. Resolution of changes is usually in the range from a few millimeters to $6 \mathrm{~cm}$ in diameter, but the changes have been reported in the literature as having a diameter of $25 \mathrm{~cm}$. Both spontaneous regression and malignancy are extremely rare. The disease is not associated with any disorder of lipid metabolism [34].
- Gardner syndrome - lipomatosis in the form of tumors or nodules on the trunk, with the presence of polyps in the intestines and the ability to form cysts and osteomas, genetically determined disease, autosomal dominant [35].

- Dercum's disease or painful obesity - usually occurs in middle-aged women more than five times more often than in men, characterized by the presence of irregular, painful lipomas placed on the torso, shoulders, arms, forearms and lower extremities. The disease normally is associated with obesity, increased tendency to develop bruises, sleep disorders and/or depression [36].

- Encephalocraniocutaneus lipomatosis (ECCL, Haberland syndrome) is a rare disease characterized by changes in the dermis and subcutaneous tissue of the face and neck, the mesenchymal tissues of the head, the bones of the skull. Asymmetrically surrounding lipomas are mentioned to co-occur with ophthalmic and neurological malformations. There are skin lesions and alopecia areata. About two-thirds of the patients presented normal physical and psychological development or mild mental retardation, epilepsy occurs in half of them [37].

The disease causes mainly aesthetic symptoms, in advanced cases, causes dysfunction of the motor system causing compression syndrome or vena cava syndrome, dysphagia, respiratory disorders, obstructive sleep apnea. Moreover, the literature includes at least 3 cases of malignant transformation of the disease in the direction of liposarcoma [9, 22].

Madelung's disease coincides with polyneuropathy, metabolic disorders, malignancies of the mouth and throat, but it is not clear whether the effects of these disorders have pathogenesis of the disease or it is a coincidence in patients in the risk group - diabetes, lipid disorders, alcoholism and smoking. Other metabolic disorders associated with MSL are insulin resistance, hyperuricemia, hypertriglyceridemia, distal tubular acidosis, increased liver enzyme levels, abnormal hormone levels of thyroid, adrenal and pituitary glands; and paradoxically, high levels of $\operatorname{HDL}[9,19,38]$.

Prognosis depends mainly on location, although some researchers indicate that depending on the severity of accompanying neuropathy, perhaps in $25.8 \%$ of cases it may be fatal [4].

There are no clear guidelines to provide treatment of patients with Madelung's disease. Changes in eating habits and reducing alcohol consumption are positive for general health, but do not resolve the changes and do not cause them to stop growing. The only effective treatment is surgical removal of the tissues.

Because of poorly understood etiology, full or causal treatment is not possible. In view of the suspicion of the essential role in pathogenesis of disorders associated with adrenergic stimulation, treatment with salbutamol were unsuccessful [39]. The disease is usually accompanied by significant hyperlipidemia, fibrate therapy 
aimed at lowering triglycerides (TG) concentrations in blood serum helps to reduce further progress of the disease. A possible suppressing mechanism of fibrates for growth activity of cells in brown adipose tissue has been described [40]. Dietary treatment did not produce the desired results, but it is advisable to improve the overall health of the patient. It is said that abstinence allows certain patients to stop the growth of tissues without affecting the regression.

The mesotherapy procedure in aesthetic surgery used for modeling of the figure, even though some active substances used in mesotherapy cocktails, such as lecithin affecting the $\beta$-adrenergic stimulation of lipolysis failed to achieve satisfactory results using this method. The use of enzymatic lipolysis gives a substantial, although short effect of reducing fat masses [41, 42].

It seems that the best methods are the methods of surgical treatment - surgical removal, or liposuction with or without USG control.

The following factors are of decisive importance for the choice of the surgical method - the location, local severity, infiltration of the surrounding structures and above all the experience and preference of the operating team $[43,44]$. Satisfactory results of surgical treatment to improve the aesthetic and functional aspects, do not exclude the possibility of recurrence.

Madelung's disease is a chronic progressive disease, and it is not easy to anticipate changes in the course of growth. It has not only an aesthetic dimension, but it often significantly affects the functioning of the patient and may even develop life-threatening consequences. The data gathered from the literature indicate that most likely etiology of the disease is multifactorial. The disorder of the mechanism of adrenergic transduction in our latitude is the trigger factor, caused by alcohol abuse in patients predisposed to the disorders of cell proliferation in residual fetal adipose tissue, comprising abnormal mitochondria and induce the chain of changes leading to the development of the disease.

Diagnostic examination should consist of a thorough clinical examination and analysis of the patient's medical history, USG of changes, CT or MRI and necessary biopsy of changes. The most important diagnosis to be taken into consideration in the differential diagnosis include: liposarcoma, multiple congenital lipomatosis, Dercum's disease, neurofibromatosis and lipomatosis induced by drugs (corticosteroids and proteases inhibitors).

Madelung's disease should be treated surgically by specialists in the area in which the changes occur, i.e. plastic surgeons, ear, nose and throat physician (ENT), thoracic surgeons or general surgeons. High tumescent PAL due to cervical manifestations of the Madelung's disease in the form of so-called Madelung's collar gives very good functional and aesthetic results and indicates that in some cases, this safe, simple and cheap method may be useful to remove large fatty deposits.

\section{Conflict of interest}

The authors declare no conflict of interest.

\section{References}

1. Brodie BC. Clinical lectures on surgery. In: Delivered at St Georges Hospital. Lea and Blanchard, Philadelphia, 1846.

2. Madelung OW. Über den Fetthals. Archiv für klinische Chirurgie 1888; 37: 106-30.

3. Launois PE Bensaude R. L'adenolipomatose symmetrique. Bulletin et Memoires de la Societe Medicale des Hospitaux des Paris 1898; 1: 298-318.

4. Zeitler H, Ulrich-Merzenich G, Richter DF, et al. Multiple benign symmetric lipomatosis - a differential diagnosis of obesity. Is there a rationale for fibrate treatment? Obes Surg 2008; 18: 240-2.

5. Sia KJ, Tang IP, Tan TY. Multiple symmetrical lipomatosis: case report and literature review. J Laryngol Otol 2012; 126: 756-8.

6. Nisoli E, Regianini L, Briscini L, et al. Multiple symmetric lipomatosis may be the consequence of defective noradrenergic modulation of proliferation and differentiation of brown fat cells. J Pathol 2002; 198: 378-87.

7. Fernández Sanromán J, Díaz Gonzalez F, Rodriguez Campo FJ. Benign symmetrical lipomatosis with mediastinal involvement and growth retardation. J Oral Maxillofac Surg 1992; 50: 299-301.

8. Larsson NG, Tulinius MH, Holme E, Oldfors A. Pathogentic aspects of the A8344G mutation of mitochondrial DNA associated with MERRF syndrome and multiple symmetric lipomas. Muscle Nerve 1995; 3: 102-6.

9. González-Garciá R, Rodríguez-Campo FJ, Sastre-Pérez J, et al. Benign symmetric lipomatosis (Madelung's disease): case reports and current management. Aesthetic Plast Surg 2004; 28: 108-12.

10. Hadjiev B, Stefanova P, Shipkov C, et al. Madelung disease: on the morphologic criteria for diagnosis and treatment. Ann Plast Surg 2010; 64: 807-8.

11. Tremp M, Wettstein R, Tchang LA, et al. Power assisted liposuction (PAL) of multiple symmetric lipomatosis (MSL) - longitudinal study. Surg Obes Relat Dis 2015; 11: 155-60.

12. Jang HJ, Lee A, Han SA, et al. Multiple symmetric lipomatosis (Madelung's disease) presenting as bilateral huge gynecomastia. J Breast Cancer 2014; 17: 397-400.

13. Tekin A, Ogetman Z. Central form of multiple symmetric lipomatosis: a case report. Cases J 2009; 12: 8427.

14. Ardeleanu V, Chicos S, Georgescu C, Tutunaru D. Multiple benign symmetric lipomatosis - a differential diagnosis of obesity. Chirurgia (Bucur) 2013; 108: 580-3.

15. Becker-Wegerich P, Steuber M, Olbrisch R, et al. Defects of mitochondrial respiratory chain in multiple symmetric lipomatosis. Arch Dermatol Res 1998; 290: 652-5.

16. Sokolov M, Mendes D, Ophir D. Madelung's disease. Isr Med Assoc J 2010; 12: 253-4.

17. Almeida FS, Pialarissi PR, Silva SL, et al. Surgical approach in a patient with multiple symmetrical lipomatosis: case report. Braz J Otorhinolaryngol 2012; 78: 142.

18. Kobayashi J, Nagao M, Miyamoto K, Matsubara S. MERRF syndrome presenting with multiple symmetric lipomatosis in a Japanese patient. Intern Med 2010; 49: 479-82.

19. Lee HW, Kim TH, Cho JW, et al. Multiple symmetric lipomatosis: Korean experience. Dermatol Surg 2003; 29: 235-40. 
20. Mevio E, Sbrocca M, Mullace M, et al. Multiple symmetric lipomatosis: a review of 3 cases. Case Rep Otolaryngol 2012; 2012: 910526.

21. Josephson GD, Sclafani AP, Stern J. Benign symmetric lipomatosis (Madelung's disease). Otolaryngol Head Neck Surg 1996; 115: 170-1.

22. Borriello M, Lucidi A, Carbone A, et al. Malignant transformation of Madelung's disease in a patient with a coincidental diagnosis of breast cancer: a case report. Diagn Pathol 2012; 7: 116 .

23. Meningaud JP, Pitak-Arnnop P, Bertrand JC. Multiple symmetric lipomatosis: case report and review of the literature. J Oral Maxillofac Surg 2007; 65: 1365-9.

24. Hadjiev B, Stefanova P, Shipkov C, et al. Madelung disease: on the morphologic criteria for diagnosis and treatment. Ann Plast Surg 2010; 64: 807-8.

25. Lopez-Ceres A, Aguilar-Lizarralde Y, Villalobos Sanchez A, et al. Benign symmetric lipomatosis of the tongue in Madelung's disease. J Craniomaxillofac Surg 2006; 34: 489-93.

26. Kontzoglou K, Stamatakos M, Polyzou E, et al. Dermatofibrosarcoma: a rare form of soft tissue. Management and review of the literature. Chirurgia (Bucur) 2011; 106: 653-6.

27. Pirvulescu I, Gheorghe L, Csiki I, et al. Noninvasive clinical model for the diagnosis of nonalcoholic steatohepatitis in overweight and morbidly obese patients undergoing bariatric surgery. Chirurgia (Bucur) 2012; 107: 772-9.

28. Kushner RF, Weinsier RL Evaluation of the obese patient. Practical considerations. Med Clin North Am 2000; 84: 387-99.

29. Kumar S P, Kamath SM, Prasad AL, et al. Acute-onset of multiple painful nodules over forearms and back. J Clin Diagn Res 2013; 7: 2314-6.

30. Szudek J, Evans DG, Friedman JM. Patterns of associations of clinical features in neurofibromatosis 1 (NF1). J Hum Genet 2003; 112: 289-97.

31. Choi HJ, Lee YM, Lee JH, et al. Pediatric lipoblastoma of the neck. J Craniofac Surg 2013; 24: e507-10.

32. Furlong MA, Fanburg-Smith JC, Miettinen M. The morphologic spectrum of hibernoma: a clinicopathologic study of 170 cases. Am J Surg Pathol 2001; 25: 809-14.

33. Rangasami R, Jyotsna M, Anupama C, Santhosh J. Congenital infiltrating lipomatosis of face. Indian J Radiol Imaging 2008; 18: 306-9.

34. Toy BR. Familial multiple lipomatosis. Dermatol Online J 2003; 9: 9.

35. Rammohan A, Wood JJ. Desmoid tumour of the breast as a manifestation of Gardner's syndrome. Int I Surg Case Rep 2012; 3: 139-42.

36. Hansson E, Svensson H, Brorson H. Review of Dercum's disease and proposal of diagnostic criteria, diagnostic methods, classification and management. Orphanet J Rare Dis 2012; 7: 23

37. Kalyan K, Vijayalakshmi B, Srinivas D, Ramanareddy G. Encephalocraniocutaneous lipomatosis (Haberl and syndrome): a case report and review of literature. J Dermatol 2013; 58: 232-4

38. Verna G, Kefalas N, Boriani F, et al. Launois-Bensaude syndrome: an unusual localization of obesity disease. Obes Surg 2008; 18: 1313-7.

39. Leung NW, Gaer J, Beggs D, et al. Multiple symmetric lipomatosis (Launois-Bensaude syndrome): effect of oral salbutamol. Clin Endocrinol (Oxf) 1987; 27: 601-6.

40. Tong Y, Hara A, Komatsu M, et al. Suppression of muscle associated proteins PPAR alpha in brown adipose tissue. Biochem Biophys Res Commun 2005; 336: 76-83.
41. Hasegawa T, Matsukura T, Ikeda S. Mesotherapy for benign symmetric lipomatosis. Aesthetic Plast Surg 2010; 34: 153-6.

42. Rotunda AM, Kolodney MS. Mesotherapy and phosphatidylcholine injections: historical clarification and review. Dermatol Surg 2006; 32: 465-80.

43. Kovács A, Klein C. Madelung disease. A case report with special reference to therapy. Chirurg 1997; 68: 276-9.

44. Zielińska-Kaźmierska B, Lewicki M, Manowska B. Madelung disease. Adv Dermatol Allergol 2015; 32: 400-3. 